\title{
Diagnostic and Therapy of Nonalcoholic Fatty Liver Disease: A Narrative Review
}

\author{
Elke Roeb \\ Gastroenterology, Justus Liebig University Giessen, Giessen, Germany
}

\section{Keywords}

Nonalcoholic fatty liver disease - Diagnosis - Therapy ·

Bariatric surgery · Diabetes · Fatty liver

\begin{abstract}
Background: The prevalence of nonalcoholic fatty liver disease (NAFLD) is increasing and strongly associated with the metabolic syndrome, especially with obesity. A subtype, nonalcoholic steatohepatitis (NASH), might progress to advanced fibrosis and cirrhosis. NASH patients have an increased all-cause mortality. First and foremost are malignancies, followed by cardiovascular diseases. Summary: The NAFLD fibrosis score and noninvasive liver stiffness measurement (transient hepatic elastography) are essential components for the diagnostic risk assessment of NAFLD patients. Other steatoses (alcohol, genetic disorders, drugs, toxins, malnutrition, etc.) must be considered in the differential diagnosis. So far, there is no approved liver-specific drug therapy with a proven effect on NAFLD for patients without diabetes mellitus. Obeticholic acid (FXR agonist), cenicriviroc (a dual inhibitor of the chemokine receptors (CCR), CCR2 and CCR5), acetyl-CoA carboxylase inhibitors, and several thyroid hormone analogs are the most advanced substances in clinical development in ongoing phase 2 and 3 studies. Key Messages: Weight loss, physical training, and the screening and treatment of risk factors represent the cornerstones of NAFLD therapy. Treatment with glucagon-like peptide 1 analogs (e.g., liraglutide, semaglutide) and sodium-dependent glucose transporter 2 inhibitors can be recommended in patients with diabetes and NASH. @2021 The Author(s)

Published by S. Karger AG, Basel
\end{abstract}

\section{Introduction}

According to the current guidelines of the DGVS, European Association for the Study of the Liver (EASL), American Association for the Study of Liver Diseases (AASLD), Asian Pacific Association for the Study of the Liver (APASL), and World Gastroenterology Organization the nonalcoholic fatty liver disease (NAFLD) includes fatty liver or nonalcoholic fatty liver (NAFL), nonalcoholic steatohepatitis (NASH), NASH fibrosis, and NASH cirrhosis. New nomenclatures (e.g., metabolically associated fatty liver disease) have so far not been able to establish themselves internationally [1]. The progression of NASH is associated with liver cell stress, consecutive inflammation, and fibrosis, with the potential development of liver cirrhosis, portal hypertension, and the socalled end-stage liver disease. NASH also displays a relevant risk factor for the occurrence of hepatocellular carcinoma (HCC) and in rare cases of intrahepatic cholangiocarcinoma. The pathogenesis and natural course of NAFLD are increasingly better understood, even if the heterogeneity of affected patients and the multifactorial genesis make it difficult to assess an individual prognosis. NASH-associated end-stage liver disease is expected to represent the highest proportion of patients listed for liver transplantation in the future [2]. The disease - although genetic factors have also been identified - is viewed as a result of hyperalimentation and the hepatic manifestation of what is known as the metabolic syndrome [3]. The clinical symptoms of noncirrhotic NAFLD are usually nonspecific. With a global prevalence of around 25\%, NAFLD is now the leading cause of chronic liver disease worldwide and a growing public health chal-

Correspondence to:

Elke Roeb, elke.roeb @innere.med.uni-giessen.de 
lenge. A further increase in NAFLD in the sense of the obesity epidemic, especially among adolescents and younger patients, is to be expected. Changes in lifestyle, demographic change, and the increasing complexity of pharmacological therapies cause an increase in the prevalence of NAFLD. Doctors and patient organizations have to deal with it collectively and individually [4].

\section{NAFLD Diagnosis}

Transabdominal ultrasound (US) should be used as primary imaging in patients with suspected NAFLD, but does not exclude hepatic steatosis and does not differentiate between NAFL and NASH [5]. US is a widely available, cost-effective, and radiation-free method that allows the diagnosis of fatty liver disease when the liver parenchyma presents with increased echogenicity. In the case of a higher degree of obesity, there is also a dorsal weakening of the parenchymal signal. Therefore, US is suitable as a screening method for determining fatty liver disease $[5,6]$. Reference is made here to S3 guidelines HCC [6, 7], the EASL-EASD-EASO Clinical Practice Guideline NAFLD, and current reviews $[8,9]$.

For the safe use of US diagnostics, knowledge of sound physics and device configuration is required. The examination should therefore be carried out under the guidance of or by experienced examiners. Under these conditions, US has an excellent specificity (>95\%) for the detection of advanced fatty liver disease [5], but the sensitivity is insufficient for minor changes (e.g., for steatosis degree S1 65\%) [10]. It is therefore not possible to exclude hepatic steatosis using US. An exact, noninvasive determination of the degree of steatosis is not possible using controlled attenuation parameter (CAP) [11]. In the case of severe obesity, a critical interpretation of the findings is necessary. The CAP technology can be used in connection with the liver stiffness measurement for the current assessment of the extent of fatty liver disease. The routine clinical use of CAP in NAFLD diagnostics cannot currently be recommended due to the limited amount of data [11].

Magnetic resonance-based procedures (MR-PDFF, MR-S) can be performed to measure fat in the liver quantitatively. Computed tomography (CT) should not be used in the primary diagnosis of NAFLD because of its radiation exposure $[12,13]$. In terms of method, however, CT is a highly reproducible and objective imaging method for displaying the fat content of the liver. Fatty liver disease can be diagnosed by means of multiparametric comparisons of the signal weakening of the parenchyma in native CT. For example, the attenuation of the parenchymal signal of $>10$ Hounsfield units compared to the spleen is a suitable diagnostic criterion.
In several comparative studies, MR-PDFF showed the highest sensitivity and specificity of all noninvasive methods for the detection of hepatic steatosis [14] and is currently the only method that can reliably noninvasively graduate the extent of steatosis in NAFLD. It should be noted that the information is based on the relative triglyceride content but cannot provide any information about the histological distribution [14]. Due to the diagnostic precision, MR-based methods appear to be suitable as a reference standard for diagnostic and interventional studies [15]. However, the clinical significance is currently limited to centers due to the low availability and the requirements for hardware and software.

\section{Diagnostic Algorithm}

For the primary clarification of high-risk patients (e.g., with obesity, type 2 diabetes [T2D] or metabolic syndrome) in whom the diagnostic imaging (e.g., using US) has shown fatty liver or who have elevated liver values (GOT, GPT, and/or $\gamma \mathrm{GT}$ ) and for whom NAFLD is suspected after other causes have been ruled out, noninvasive fibrosis scores such as FIB-4 [16] or NAFLD fibrosis score (NFS) are suitable for risk assessment $[17,18]$ (Fig. 1). Due to the high negative predictive value of FIB4 or NFS ( $\geq 90 \%)$ and taking into account the respective low limit value (FIB- $4 \leq 1.3$ or NFS $<-1.455$ ), advanced fibrosis can be highly probable and be excluded [16]. In patients with a low risk of fibrosis, follow-up monitoring, for example, by FIB-4 or NFS and the transaminases, can be carried out at regular intervals.

The diagnostic value for the exclusion of advanced liver fibrosis is comparable for the NFS and FIB-4 index, although the FIB-4 is based on fewer parameters. The FIB-4 is therefore easier to identify, less expensive, and should be used as a priority.

The NFS consists of age, BMI, glucose/T2D, platelet count, albumin, and AST/ALT quotient, which can be calculated free of charge via http://nafldscore.com. In a meta-analysis of 64 studies with 13,046 patients, the diagnostic accuracy (AUC) of the NFS for the diagnosis of advanced fibrosis $(F \geq 3)$ was 0.84 [19]. The NFS encompasses a low and high limit value of $<1.455$ and $>0.676$. With an NFS of $<-1.455$, advanced fibrosis could be excluded with a sensitivity of $82 \%$ (exploration cohort, $n=480$ ) or $77 \%$ (validation cohort, $n=253$ ) and a negative predictive value of $93 \%$ or $88 \%$ [20]. The FIB4 Index is a test that can be calculated free of charge at http://gihep.com/calculators/hepatology/fibrosis4-score/ and consists of age, platelet count, AST, and ALT. For the FIB-4 index in NAFLD, a low and high limit value of 1.3 and 2.67 was identified for clarifying advanced fibrosis [21]. Others describe a cutoff of 1.3 as inappropriate because of including patients with F2 fibrosis [22]. 


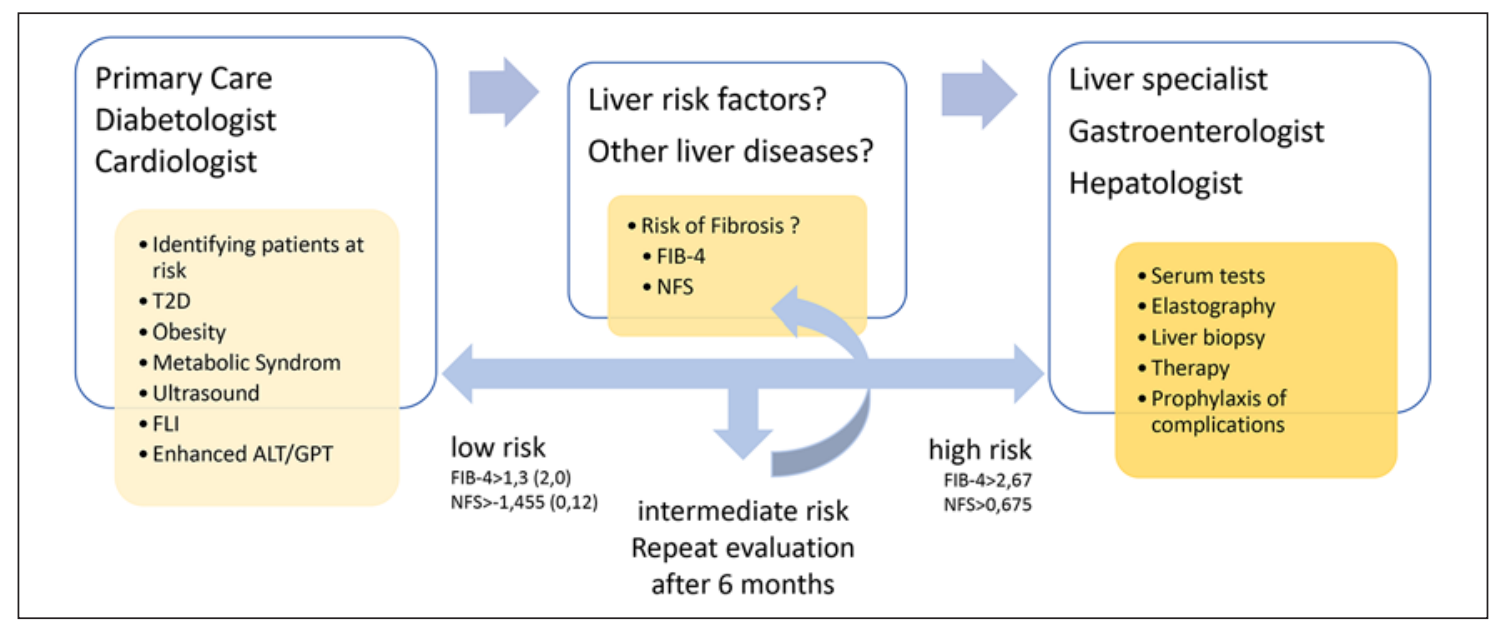

Fig. 1. Diagnostic of NAFLD. FLI; NFS; and T2D. This algorithm corresponds well to the so-called European algorithm of the EASLEASD-EASO Clinical Practice Guidelines and a recently proposed procedure for general practitioners and diabetologists [18] but is simpler in handling. The sequence of FLI and FIB-4 is dedicated to screening in a risk group with T2D. The use of age-adjusted limit values (in brackets) is recommended for FIB- 4 and NFS to reduce the high proportion of intermediate tested persons. NAFLD, nonalcoholic fatty liver disease; NFS, NAFLD fibrosis score; EASL, European Association for the Study of the Liver; T2D, type 2 diabetes; FLI, fatty liver index.
In patients with FIB- 4 or NFS in the intermediate (between the 2 limit values) or high (FIB- $4 \geq 2.67$ or NFS $>0.676$ ), an elastography using vibration-controlled transient elastography (VCTE; FibroScan ${ }^{\circledR}$ ) is used as an additional test method. However, taking relevant comorbidities into account is recommended [17, 18]. Alternatively, shear wave-based elastography methods can be used, whereby the manufacturer's specific cutoffs must be taken into account [23]. Shear wave elastography methods are available as software components for many modern US devices and can therefore be easily used when performing an abdominal US. Compared to the VCTE, the shear wave elastography procedures are less well evaluated for the risk stratification of NAFLD and are not yet included in the current recommendations of international specialist societies for clarifying NAFLD (AWMF/ DGVS 2015, EASL Guideline 2016, AASLD Practice Guidance NAFLD 2018) $[8,17,24]$. A potential diagnostic algorithm is shown in Figure 1.

In summary, risk stratification should be carried out in all patients with regard to the underlying fibrosis stage when NAFLD is diagnosed for the first time. For this purpose, noninvasive tests/scores (NFS, FIB-4) or elastography (VCTE), possibly also in combination, should be used. Control intervals for noninvasive test procedures should be based on the initial findings. US-based elastography methods can be used to rule out advanced liver fibrosis and liver cirrhosis in NAFLD. A liver biopsy should be performed if fibrosis is to be reliably detected or ruled out. Note that patients with noninvasive or histological evidence of cirrhosis should be monitored regularly for the development of liver-related complications.

\section{Therapy}

A reduction in body weight in overweight or obese NAFLD patients is accompanied by regression of steatosis $[25,26]$. The decrease in steatosis and ALT is proportional to the weight loss; there is a clear relationship between dose and effect [27]. And it is entirely irrelevant in which way the weight loss was achieved $[26,28]$.

To answer the question of regression of an already existing NASH cirrhosis or the prevention of disease progression with the development of HCC, no results from studies on lifestyle intervention are available so far. All in all, a weight reduction of at least $10 \%$ is extremely effective in the treatment of NASH ( $90 \%$ cure rate), but in clinical practice a goal that was only achieved by $10 \%$ of patients [29].

Meta-analyzes show that aerobic training and/or isometric training in NAFLD patients also improved transaminases and hepatic fat content independently of weight loss [30,31]. Both training concepts are apparently equally effective. Overweight or obese NAFLD patients should be recommended to lose weight by means of hypocaloric nutrition in accordance with the recommendations of the DAG, S3 Guideline on Obesity (AWMF 050-001). A Mediterranean diet might be considered [32].

In NAFLD people of normal weight (Lean NAFLD), regardless of weight loss, physical activity should be aimed for in accordance with the age-adapted WHO recommendations with the aim of building muscle. A Mediterranean diet can also be recommended for NAFLD people of normal weight (Lean NAFLD) [33]. 
The rapidly increasing prevalence of obesity over the past few decades has been associated with the increasing consumption of fructose and fructose-containing corn syrup in processed foods and beverages [34-36]. However, meta-analyzes did not show that fructose consumption in the context of a normo-caloric diet favors the development or progression of NAFLD [37, 38]. In a double-blind study in overweight people, excessive calorie intake, but not fructose compared to isocaloric amounts of glucose, was associated with an increase in the hepatic fat content and transaminases [39].

Systematic reviews and meta-analyzes suggest that coffee drinking leads to a reduction in the risk of HCC. Higher doses of coffee resulted in a higher risk reduction $[40,41]$. However, increased coffee consumption is not associated with a reduced risk of hepatobiliary carcinoma [42]. The protective agents from coffee and the molecular mechanisms of HCC prevention remained unclear so far.

Positive effects with regard to coffee consumption can be derived from epidemiological studies $[43,44]$. These showed a protective effect of coffee consumption in relation to the risk of suffering from NAFLD and also in relation to the fibrosis stage. To date, no controlled studies are available on this subject. In a pooled meta-analysis with a total of 11 studies, people who drank coffee had a relative risk of 0.77 (95\% CI $0.60-0.98$ ) of suffering from NAFLD. In addition, there is also a significantly reduced risk of advanced liver fibrosis compared to patients who do not drink any coffee (RR 0.68 (95\% CI 0.68-0.79) [44].

Up to now, there are no approved medications for the indication NAFLD. The general use of drugs such as ursodeoxycholic acid, pioglitazone, metformin, silymarin, or pentoxifylline as well as dietary supplements such as vitamin E or omega-3 fatty acids should not take place due to the current data on the treatment of NAFLD. Due to the beneficial effects on NASH, noncirrhotic NAFLD patients with T2D should use metformin plus glucagonlike peptide 1 (GLP-1) agonists (e.g., liraglutide or semaglutide). The use of sodium-dependent glucose transporter 2 (SGLT2) inhibitors, for example, empagliflozin and dapagliflozin, or the thiazolidinedione pioglitazone may be considered in these patients as well.

Patients with NASH-associated liver cirrhosis and T2D with compensated cirrhosis of the liver in Child A stage and normal kidney function are allowed to receive metformin. There is currently insufficient experience with the possible use of GLP-1 agonists, SGLT2 inhibitors, or pioglitazone in patients with $\mathrm{NASH}$-associated liver cirrhosis.

Other antidiabetic agents, such as metformin, dipeptidyl peptidase IV inhibitors, or insulin, have shown no specific advantages with regard to NAFLD therapy so far. However, large retrospective studies have reported that metformin in NAFLD patients has a reduced risk of devel-
Physical activity \& weight loss (hypocaloric nutrition)

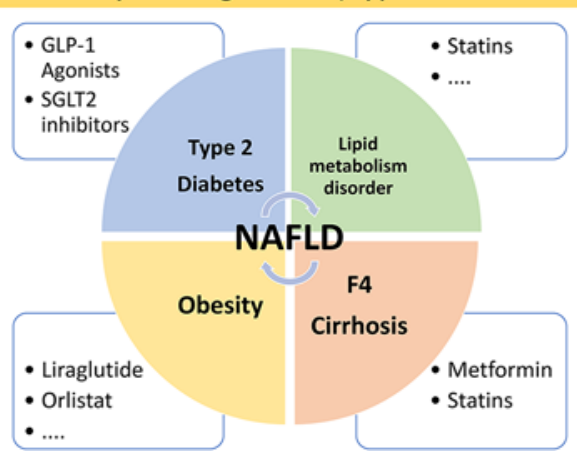

Fig. 2. Therapy of NAFLD with or without comorbidities. The base of NAFLD therapy is the so-called lifestyle modification. Overweight or obese NAFLD patients should be recommended to lose weight by means of hypocaloric nutrition. Aerobic training and/or isometric training in NAFLD patients improves transaminases and hepatic fat content independently of weight loss. Comorbidities should be treated as recommended. NAFLD, nonalcoholic fatty liver disease.

oping HCC [45]. Even in patients with NASH-associated compensated cirrhosis of the Child A stage, the use of metformin for the treatment of diabetes is associated with a reduced risk of hepatic decompensation and HCC (a dose of $2 \mathrm{~g}$ per day with normal kidney function can be used) $[46,47]$. Metformin is not allowed if the GFR is below 30 $\mathrm{mL} / \mathrm{min}$. However, there are no prospective controlled studies on the use of metformin in liver cirrhosis to date.

A placebo-controlled study of patients with NASH and T2DM showed a greater reduction in liver fat content for vitamin $\mathrm{E}$ (800 IU/day) and a more frequent reduction in NASH without improvement in fibrosis [48]. The risk of increased mortality and morbidity with vitamin E supplementation limits its use, particularly in patients with T2D.

Because lipid metabolic disorders, such as familial hypercholesterolemia, hypertriglyceridemia, lipoprotein (a) elevation, or isolated HDL cholesterol lowering, represent a greatly increased risk of cardiovascular diseases, and NAFLD might increase the risk of cardiovascular diseases regardless of a lipid metabolism disorder, the lipid metabolism disorder should be effectively treated [49, 50].

If obesity is present in NAFLD patients, it should be treated effectively as well. Due to the favorable effects on $\mathrm{NASH}$, in noncirrhotic NAFLD patients with obesity and an indication for medicinal weight reduction, GLP-1 agonists, for example, liraglutide, can be used. Orlistat, which is approved for the treatment of obesity, can be used in overweight and obese patients with NASH in almost the same manner. Current treatment strategies are summarized in Figure 2. 
A number of substances are currently being investigated in clinical phase 3 and phase 2 studies whose mechanisms of action lie in the pathophysiological processes of glucose metabolism, the inhibition of de novo lipogenesis, inflammation, or fibrogenesis. These substance classes include agonists of the nuclear receptors FXR (or its action mediator fibroblast growth factor/FGF19) and peroxisome proliferator-activated receptors (PPAR), chemokine receptor (CCR) inhibitors, thyroid hormone receptor- $\beta$ agonists, inhibitors of lipogenic key enzymes such as FASN and SCD-1, and enterohepatic hormones and their agonists, such as GLP-1, FGF19, or FGF21. Medicines with a primarily antidiabetic effect, such as the group of SGLT2 inhibitors, should also be mentioned here.

Obeticholic acid (FXR agonist), resmetirom (thyroid hormone receptor- $\beta$ agonist), and aramchol (SCD-1 inhibitor) are currently in phase 3 (for review see Roeb and Geier [51]). Only for obeticholic acid are positive data available from an interim analysis with regard to fibrosis improvement as a co-primary endpoint (REGENERATE study) [52]. Several substances did not produce positive results in phase 3 and are therefore no longer being developed as individual substances in this indication, namely elafibranor (PPAR $\alpha / \delta$ agonist), cenicriviroc (CCR2/5 inhibitor), and selonsertib (ASK1 inhibitor). Further FXR agonists (Tropifexor, Cilofexor), recombinant FGF19 (Aldafermin), different variants of FGF21 (Pegbelfermin, Efruxifermin), GLP1 analogs (liraglutide, semaglutide), and pan-PPAR agonists (Lanifibranor) showed promising results in phase 2 studies. According to the current status, obeticholic acid is the only drug with a significant benefit on fibrosis in the phase 3 interim analysis, the primary candidate for the first conditional approval; however, this was not granted at the time this review was published [53].

For the future, more "personalized" therapy concepts are to be expected. This might include the targeted "correction" of the intestinal microbiota to reduce NAFLD and cardiometabolic comorbidities [54] or targeted therapies based on genetic risk stratification. The classic example of this would be the single nucleotide polymorphism in the patatin-like phospholipase domain-containing 3 gene (PNPLA3), rs738409, which codes for the missense mutation I148M. Targeted drugs (e.g., antisense oligonucleotides, tyrosine kinase inhibitor momelotinib) could inhibit PNPLA3 levels in 148M homozygous persons and thus a pathomechanism for progression [55].

Bariatric surgery has proven to be the most effective therapy for morbid obesity. Furthermore, obesity surgical operations usually lead to an improvement and often also a complete remission of obesity-associated secondary diseases [56]. According to the current German S3 guideline of the DGAV from 2018, obesity surgery is indicated in the presence of severe obesity with a BMI $\geq 40 \mathrm{~kg} / \mathrm{m}^{2}$ (even without concomitant diseases) if conservative measures for weight reduction (diet change, exercise measures, and, if necessary, behavioral therapy) have failed. In addition, this should be offered with a BMI $\geq 35 \mathrm{~kg} / \mathrm{m}^{2}$ and at least 1 major obesity-specific concomitant disease, such as NAFLD and NASH, if conservative measures for weight reduction have failed [56].

It should be noted that NAFLD and NASH are frequent comorbidities in obese patients. Various studies and a current meta-analysis show that bariatric surgery led to high remission rates of NAFLD and NASH in these patients. The qualitatively best data come from the "Lille Bariatric Cohort," which showed a high remission rate for NASH over 5 years with histologically confirmed NASH. The study also showed that bariatric surgery can lead to long-term improvement in existing liver fibrosis, even if the fibrosis progressed in a small percentage [56].

Before listing for LTX, a multidisciplinary evaluation of the patients should be carried out due to the increased perioperative risk, especially with regard to the increased occurrence of cardiovascular events and infectious complications. According to LTX, the increased risk of recurrence of metabolic syndrome, cardiovascular disease, and recurrence of NAFLD should be taken into account in the patient management as well as in the selection of immunosuppressive medication.

Note that in obesity and NAFLD sleeve gastrectomy, Roux-Y gastric bypass and single-anastomotic gastric bypass can be performed as metabolic surgical procedures. The adjustable gastric band should not be used in obesity and NAFLD because of its inferior effectiveness $[56,57]$.

Because of the risk of progressive liver failure, the severity of NAFLD should be considered critically when malabsorptive procedures are indicated (e.g., biliopancreatic diversion, distal gastric bypass, and single-anastomotic bypass with a biliopancreatic loop $>200 \mathrm{~cm}$ long). In the presence of liver cirrhosis, sleeve gastrectomy should preferably be used.

\section{Conclusion}

An established drug therapy for NAFLD, especially $\mathrm{NASH}$, is not (yet) available. Besides the treatment of comorbidities (diabetes, lipid metabolism disorders, and obesity), regular physical endurance activity (endurance sports such as cycling, walking, or swimming for at least $3 \times 45$ min per week) and an optimized diet (reduction in calorie intake) constitute the best recommendation. The aim is to slowly reduce body weight. This might also have a positive effect on the accompanying diseases, such as T2D and obesity. Reduction in body weight is also able to reduce the inflammatory activity in the liver tissue. 


\section{Conflict of Interest Statement}

The authors received fees from the following companies: Abbvie, Bristol Myers Squibb, Falk Foundation, Gilead, Intercept, Merz, Norgine, and Pfizer for lectures and advice. There is no conflict of interest with regard to this review.

\section{Funding Sources}

This work was supported by grants from the German Research Foundation (RO957/10-1, RO3714/4-1), the von-Behring-Roentgen Foundation (\#66-0008), and the University Hospital Giessen and Marburg (UKGM).

\section{References}

1 Roeb E. Excess body weight and metabolic (dysfunction)-associated fatty liver disease (MAFLD). Visc Med. 2021:37:273-80.

2 Younossi ZM. Non-alcoholic fatty liver disease - a global public health perspective. J Hepatol. 2019 Mar;70(3):531-44.

3 Alberti KG, Eckel RH, Grundy SM, Zimmet PZ, Cleeman JI, Donato KA, et al. Harmonizing the metabolic syndrome: a joint interim statement of the International Diabetes Federation Task Force on epidemiology and prevention; National Heart, Lung, and Blood Institute; American Heart Association; World Heart Federation; International Atherosclerosis Society; and International Association for the Study of Obesity. Circulation. 2009 Oct 20;120(16):1640-5.

4 Estes C, Anstee QM, Arias-Loste MT, Bantel $\mathrm{H}$, Bellentani S, Caballeria J, et al. Modeling NAFLD disease burden in China, France, Germany, Italy, Japan, Spain, United Kingdom, and United States for the period 20162030. J Hepatol. 2018 Oct;69(4):896-904.

5 Ballestri S, Nascimbeni F, Lugari S, Lonardo A, Francica G. A critical appraisal of the use of ultrasound in hepatic steatosis. Expert Rev Gastroenterol Hepatol. 2019 Jul;13(7):66781.

6 Loomba R, Lim JK, Patton H, El-Serag HB. AGA clinical practice update on screening and surveillance for hepatocellular carcinoma in patients with nonalcoholic fatty liver disease: expert review. Gastroenterology. 2020 May;158(6):1822-30.

7 Greten TF, Malek NP, Schmidt S, Arends J, Bartenstein P, Bechstein W, et al. [Diagnosis of and therapy for hepatocellular carcinoma]. Z Gastroenterol. 2013 Nov;51(11):1269-326.

8 European Association for the Study of the Liver (EASL); European Association for the Study of Diabetes (EASD); European Association for the Study of Obesity (EASO). EASLEASD-EASO clinical practice guidelines for the management of non-alcoholic fatty liver disease. J Hepatol. 2016 Jun;64(6):1388-402.

9 Bengtsson B, Stål P, Wahlin S, Björkström NK, Hagström H. Characteristics and outcome of hepatocellular carcinoma in patients with NAFLD without cirrhosis. Liver Int. 2019 Jun;39(6):1098-108.

10 Kromrey ML, Ittermann T, Berning M, Kolb C, Hoffmann RT, Lerch MM, et al. Accuracy of ultrasonography in the assessment of liver fat compared with MRI. Clin Radiol. 2019 Jul; 74(7):539-46.

11 Karlas T, Petroff D, Sasso M, Fan JG, Mi YQ de Lédinghen $\mathrm{V}$, et al. Individual patient data meta-analysis of controlled attenuation parameter (CAP) technology for assessing steatosis. J Hepatol. 2017 May;66(5):102230 .
12 Tang A, Desai A, Hamilton G, Wolfson T, Gamst A, Lam J, et al. Accuracy of MR imaging-estimated proton density fat fraction for classification of dichotomized histologic steatosis grades in nonalcoholic fatty liver disease. Radiology. 2015 Feb;274(2):416-25.

13 Yokoo T, Serai SD, Pirasteh A, Bashir MR, Hamilton G, Hernando D, et al. Linearity, bias, and precision of hepatic proton density fat fraction measurements by using MR imaging: a meta-analysis. Radiology. 2018 Feb; 286(2):486-98.

14 Choi SJ, Kim SM, Kim YS, Kwon OS, Shin SK, Kim KK, et al. Magnetic resonance-based assessments better capture pathophysiologic profiles and progression in nonalcoholic fatty liver disease. Diabetes Metab J. 2020 Oct 28. Online ahead of print.

15 Caussy C, Johansson L. Magnetic resonancebased biomarkers in nonalcoholic fatty liver disease and nonalcoholic steatohepatitis. Endocrinol Diabetes Metab. 2020 Oct;3(4): e00134.

16 Lee J, Vali Y, Boursier J, Spijker R, Anstee QM, Bossuyt PM, et al. Prognostic accuracy of FIB-4, NAFLD fibrosis score and APRI for NAFLD-related events: a systematic review. Liver Int. $2021 \mathrm{Feb}$;11(2):261-70.

17 Chalasani N, Younossi Z, Lavine JE, Charlton $\mathrm{M}$, Cusi K, Rinella M, et al. The diagnosis and management of nonalcoholic fatty liver disease: practice guidance from the American Association for the Study of Liver Diseases. Hepatology. 2018 Jan;67(1):328-57.

18 European Association for the Study of the Liver; Clinical Practice Guideline Panel; Chair; EASL Governing Board representative; Panel members. Easl clinical practice guidelines (Cpgs) on non-invasive tests for evaluation of liver disease severity and prognosis- 2021 update. J Hepatol. 2021 Sep;75(3):659-89.

19 Xiao G, Zhu S, Xiao X, Yan L, Yang J, Wu G. Comparison of laboratory tests, ultrasound, or magnetic resonance elastography to detect fibrosis in patients with nonalcoholic fatty liver disease: a meta-analysis. Hepatology. 2017 Nov;66(5):1486-501.

20 Angulo P, Kleiner DE, Dam-Larsen S, Adams LA, Bjornsson ES, Charatcharoenwitthaya $\mathrm{P}$, et al. Liver fibrosis, but no other histologic features, is associated with long-term outcomes of patients with nonalcoholic fatty liver disease. Gastroenterology. 2015 Aug; 149(2):389-97.e10.

21 Vilar-Gomez E, Calzadilla-Bertot L, Wai-Sun Wong V, Castellanos M, Aller-de la Fuente R, Metwally $\mathrm{M}$, et al. Fibrosis severity as a determinant of cause-specific mortality in patients with advanced nonalcoholic fatty liver disease: a multi-national cohort study. Gastroenterology. 2018 Aug;155(2):443-57.e17.
22 Shah S, Dhami-Shah H, Kamble S, Shukla A. FIB-4 cut-off of 1.3 may be inappropriate in a primary care referral pathway for patients with non-alcoholic fatty liver disease. J Hepatol. 2020 Jul;73(1):216-7.

23 Piscaglia F, Salvatore V, Mulazzani L, Cantisani V, Schiavone C. Ultrasound shear wave elastography for liver disease. a critical appraisal of the many actors on the stage. Ultraschall Med. 2016 Feb;37(1):1-5.

24 Roeb E, Steffen HM, Bantel H, Baumann U, Canbay A, Demir M, et al. [S2k guideline nonalcoholic fatty liver disease]. Z Gastroenterol. 2015 Jul;53(7):668-723.

25 Chalasani N, Younossi Z, Lavine JE, Diehl AM, Brunt EM, Cusi K, et al. The diagnosis and management of non-alcoholic fatty liver disease: practice guideline by the American Association for the study of liver diseases, American College of Gastroenterology, and the American Gastroenterological Association. Hepatology. 2012 Jun;55(6): 2005-23.

26 Plauth M, Bernal W, Dasarathy S, Merli M, Plank LD, Schütz T, et al. ESPEN guideline on clinical nutrition in liver disease. Clin Nutr. 2019 Apr;38(2):485-521.

27 Koutoukidis DA, Koshiaris C, Henry JA, Noreik M, Morris E, Manoharan I, et al. The effect of the magnitude of weight loss on nonalcoholic fatty liver disease: a systematic review and meta-analysis. Metabolism. 2021 Feb;115:154455.

28 Burza MA, Romeo S, Kotronen A, Svensson PA, Sjöholm K, Torgerson JS, et al. Long-term effect of bariatric surgery on liver enzymes in the Swedish Obese Subjects (SOS) study. PLoS One. 2013;8(3):e60495.

29 Romero-Gómez M, Zelber-Sagi S, Trenell M. Treatment of NAFLD with diet, physical activity and exercise. J Hepatol. 2017 Oct;67(4): 829-46.

30 Orci LA, Gariani K, Oldani G, Delaune V, Morel P, Toso C. Exercise-based interventions for nonalcoholic fatty liver disease: a meta-analysis and meta-regression. Clin Gastroenterol Hepatol. 2016 Oct; 14(10):1398411

31 Hashida R, Kawaguchi T, Bekki M, Omoto M, Matsuse H, Nago T, et al. Aerobic vs. resistance exercise in non-alcoholic fatty liver disease: a systematic review. J Hepatol. 2017 Jan; 66(1):142-52.

32 Katsagoni CN, Papatheodoridis GV, Ioannidou P, Deutsch M, Alexopoulou A, Papadopoulos $\mathrm{N}$, et al. Improvements in clinical characteristics of patients with non-alcoholic fatty liver disease, after an intervention based on the Mediterranean lifestyle: a randomised controlled clinical trial. Br J Nutr. 2018 Jul; 120(2):164-75. 
33 Zelber-Sagi S, Buch A, Yeshua H, Vaisman N, Webb M, Harari G, et al. Effect of resistance training on non-alcoholic fatty-liver disease a randomized-clinical trial. World J Gastroenterol. 2014 Apr 21;20(15):4382-92.

34 Bray GA, Nielsen SJ, Popkin BM. Consumption of high-fructose corn syrup in beverages may play a role in the epidemic of obesity. Am J Clin Nutr. 2004 Apr;79(4):537-43.

35 Jensen T, Abdelmalek MF, Sullivan S, Nadeau KJ, Green M, Roncal C, et al. Fructose and sugar: a major mediator of non-alcoholic fatty liver disease. J Hepatol. 2018 May;68(5): 1063-75.

36 Roeb E, Weiskirchen R. Fructose and nonalcoholic steatohepatitis. Front Pharmacol. 2021;12:634344.

37 Chiu S, Sievenpiper JL, de Souza RJ, Cozma AI, Mirrahimi A, Carleton AJ, et al. Effect of fructose on markers of non-alcoholic fatty liver disease (NAFLD): a systematic review and meta-analysis of controlled feeding trials. Eur J Clin Nutr. 2014 Apr;68(4):416-23.

38 Chung M, Ma J, Patel K, Berger S, Lau J, Lichtenstein AH. Fructose, high-fructose corn syrup, sucrose, and nonalcoholic fatty liver disease or indexes of liver health: a systematic review and meta-analysis. Am J Clin Nutr. 2014 Sep;100(3):833-49.

39 Johnston RD, Stephenson MC, Crossland H, Cordon SM, Palcidi E, Cox EF, et al. No difference between high-fructose and high-glucose diets on liver triacylglycerol or biochemistry in healthy overweight men. Gastroenterology. 2013 Nov;145(5):1016-25.e2.

40 Bhurwal A, Rattan P, Yoshitake S, Pioppo L, Reja D, Dellatore $\mathrm{P}$, et al. Inverse association of coffee with liver cancer development: an updated systematic review and meta-analysis. J Gastrointestin Liver Dis. 2020 Sep 9;29(3): 421-8.

41 Zhao LG, Li ZY, Feng GS, Ji XW, Tan YT, Li $\mathrm{HL}$, et al. Coffee drinking and cancer risk: an umbrella review of meta-analyses of observational studies. BMC Cancer. 2020 Feb 5;20(1): 101.
42 Godos J, Micek A, Marranzano M, Salomone F, Rio DD, Ray S. Coffee consumption and risk of biliary tract cancers and liver cancer: a dose-response meta-analysis of prospective cohort studies. Nutrients. 2017 Aug 28;9(9): 950

43 Piovani D, Danese S, Peyrin-Biroulet L, Nikolopoulos GK, Lytras T, Bonovas S. Environmental risk factors for inflammatory bowel diseases: an umbrella review of meta-analyses. Gastroenterology. 2019 Sep;157(3):64759.e4.

44 Hayat U, Siddiqui AA, Okut H, Afroz S, Tasleem S, Haris A. The effect of coffee consumption on the non-alcoholic fatty liver disease and liver fibrosis: a meta-analysis of 11 epidemiological studies. Ann Hepatol. 2021 JanFeb;20:100254.

45 Fujiwara N, Friedman SL, Goossens N, Hoshida Y. Risk factors and prevention of hepatocellular carcinoma in the era of precision medicine. J Hepatol. 2018 Mar;68(3):526-49.

46 Kaplan DE, Serper M, John BV, Tessiatore KM, Lerer R, Mehta R, et al. Effects of metformin exposure on survival in a large national cohort of patients with diabetes and cirrhosis. Clin Gastroenterol Hepatol. 2020 Aug 13. Online ahead of print.

47 Vilar-Gomez E, Calzadilla-Bertot L, Wong VW-S, Castellanos M, Aller-de la Fuente R, Eslam M, et al. Type 2 diabetes and metformin use associate with outcomes of patients with nonalcoholic steatohepatitis-related, Child-Pugh A cirrhosis. Clin Gastroenterol Hepatol. 2021 Jan;19(1):136-45.e6.

48 Bril F, Biernacki DM, Kalavalapalli S, Lomonaco R, Subbarayan SK, Lai J, et al. Role of vitamin $\mathrm{E}$ for nonalcoholic steatohepatitis in patients with type 2 diabetes: a randomized controlled trial. Diabetes Care. 2019 Aug; 42(8):1481-8.
49 Adams LA, Anstee QM, Tilg H, Targher G. Non-alcoholic fatty liver disease and its relationship with cardiovascular disease and other extrahepatic diseases. Gut. 2017 Jun;66(6): 1138-53.

50 Stefan N, Häring HU, Schulze MB. Metabolically healthy obesity: the low-hanging fruit in obesity treatment? Lancet Diabetes Endocrinol. 2018 Mar;6(3):249-58.

51 Roeb E, Geier A. Nonalcoholic steatohepatitis (NASH) - current treatment recommendations and future developments. Z Gastroenterol. 2019 Apr;57(4):508-17.

52 Younossi ZM, Ratziu V, Loomba R, Rinella M, Anstee QM, Goodman Z, et al. Obeticholic acid for the treatment of non-alcoholic steatohepatitis: interim analysis from a multicentre, randomised, placebo-controlled phase 3 trial. Lancet. 2019 Dec 14;394(10215):2184-96.

53 Mullard A. FDA rejects NASH drug. Nat Rev Drug Discov. 2020 Aug;19(8):501.

54 Acharya C, Bajaj JS. Chronic liver diseases and the microbiome-translating our knowledge of gut microbiota to management of chronic liver disease. Gastroenterology. 2021 Jan;160(2):556-72.

55 Lindén D, Ahnmark A, Pingitore P, Ciociola E, Ahlstedt I, Andréasson AC, et al. Pnpla3 silencing with antisense oligonucleotides ameliorates nonalcoholic steatohepatitis and fibrosis in Pnpla3 I148M knock-in mice. Mol Metab. 2019 Apr;22:49-61.

56 Lassailly G, Caiazzo R, Ntandja-Wandji LC, Gnemmi V, Baud G, Verkindt H, et al. Bariatric surgery provides long-term resolution of nonalcoholic steatohepatitis and regression of fibrosis. Gastroenterology. 2020 Oct;159(4): 1290-1301.e5.

57 Caiazzo R, Lassailly G, Leteurtre E, Baud G, Verkindt H, Raverdy V, et al. Roux-en-Y gastric bypass versus adjustable gastric banding to reduce nonalcoholic fatty liver disease: a 5-year controlled longitudinal study. Ann Surg. 2014 Nov;260(5):893-9. discussion 898-9. 